\title{
Cisplatin-induced apoptosis in human malignant testicular germ cell lines depends on MEKJERK activation
}

\author{
S Schweyer,', A Soruri' ${ }^{2}$, O Meschter', A Heintze', F Zschunke', N Miosge ${ }^{3}$, P Thelen ${ }^{4}$, T Schlott' $^{\prime}$, HJ Radzun' \\ and A Fayyazi'
}

'Department of Pathology, University of Göttingen, Robert-Koch-Str. 40, D-37075 Göttingen, Germany; ${ }^{2}$ Department of Immunology, University of Göttingen, Robert-Koch-Str. 40, D-37075 Göttingen, Germany; ${ }^{3}$ Department of Histology, University of Göttingen, Robert-Koch-Str. 40, D-37075

Göttingen, Germany; ${ }^{4}$ Department of Urology, University of Göttingen, Robert-Koch-Str. 40, D-37075 Göttingen, Germany

\begin{abstract}
Testicular germ cell tumours (TGCT) represent the most common malignancies in young males. Whereas in 1970 s, the survival rate in patients with metastatic testicular tumours was only $5 \%$, these days, $80 \%$ of the patients treated by modern chemotherapy will survive their disease. The drug that revolutionised the cure rate for patients with metastatic testicular tumours was cisdiamminedichloroplatinum (cisplatin, CDDP). In vitro experiments on neoplastic germ cell lines showed that their exquisite sensitivity to CDDP could be attributed to p53-dependent and -independent pathways. Applying cDNA macroarray, semiquantitative RT-PCR and Western blot analyses, blocking experiments, caspase activity assays, and morphological methods, we sought here to define the p53-independent pathway(s) involved in the CDDP-induced apoptosis. For this purpose, we used the human TGCT cell line NCCIT, the mutated p53 of which is known to remain inactive during the course of CDDP-induced apoptosis. Our experiments showed that within hours of CDDP application, two prototype members of the 'mitogen-activated protein kinase' (MAPK) family, designated 'MAPK ERK kinase' (MEK) and 'extracellular signal-regulated kinase' (ERK), were dually phosphorylated and caspase-3 became active. Functional assays using MEK inhibitors demonstrated that the phosphorylation of MEK and ERK was required for the activation of caspase-3 as the executing caspase. Interestingly, experiments with the human malignant germ cell line NTERA, which is known to possess wild-type p53, revealed the same results. Thus, our data suggest that CDDP mediates its p53independent apoptosis-inducing effect on the malignant human testicular germ cells - at least partially - through activation of the MEK-ERK signalling pathway.
\end{abstract}

British Journal of Cancer (2004) 91, 589-598. doi:10.1038/sj.bjc.6601919 www.bjcancer.com

Published online 13 July 2004

(c) 2004 Cancer Research UK

Keywords: cisplatin; apoptosis; TGCT; MEK; ERK

Except in young males, testicular germ cell tumours (TGCT) are relatively uncommon malignancies. Despite the paucity, however, TGCT have become a model for a curable neoplasm even in advanced stages. The breakthrough in the treatment of metastatic TGCT was achieved by the drug cisdiamminedichloroplatinum (cisplatin, CDDP) (Higby et al, 1974; Einhorn, 2002). Cisplatin is a potent inducer of apoptosis in different cell types and is one of the most effective and widely used chemotherapeutic agents in the treatment of human cancers. Although CDDP-induced apoptosis is known to be the result of its ability to damage DNA (Wyllie et al, 1980; Fichtinger-Schepman et al, 1985; Sorenson and Eastman, 1988; Eastman, 1990), the mechanisms by which CDDP initiates apoptosis in TGCT are not completely understood.

In mice, elevated levels of wild-type p53 in TGCT seem to contribute to their sensitivity to DNA-damaging drugs. Using the murine TGCT cell line F9, Lutzker and Levine (1996) demonstrated

\footnotetext{
*Correspondence: Dr S Schweyer;

E-mail:sswyer@med.uni-goettingen.de

Received 27 November 2003; revised 12 March 2004; accepted 26 April 2004; published online 13 July 2004
}

that a functionally inactive p53 protein in neoplastic germ cells became active following chemotherapy, thus leading to apoptosis. Although Chresta et al (1996) postulated that a hypersensitivity of human TGCT to drug-dependent apoptosis may also be associated with functional p53, Burger et al (1999) showed that abrogation of the p53 function does not affect the hypersensitivity of human TGCT cell lines to chemotherapy. Thus in human TGCT, apoptotic signalling pathways including those activated by CDDP seem to be - at least partly - p53 independent (Burger et al, 1997; Kersemaekers et al, 2002).

In the present study, we sought to define the p53-independent pathway(s) involved in the CDDP-induced apoptosis of the human TGCT cell line NCCIT, the p53 of which has been shown to remain inactive during the process of CDDP-induced apoptosis (Burger et al, 1999). Based on results from a cDNA macroarray analysis, we assumed that CDDP exploits the 'mitogen-activated protein kinase' (MAPK) signalling pathway to induce apoptosis in NCCIT cells.

Mitogen-activated protein kinase constitutes a superfamily of kinases that coordinates and transduces incoming signals from stress and growth factors, hormones and cytokines (Raabe and Rapp, 2002). The most extensively studied MAPK pathway is the MAPK kinase (MEK) - extracellular signal-regulated kinase (ERK) 
cascade, where p-MEK1/2 phosphorylates and thereby activates ERK. Originally the ERK module was thought to mediate cell proliferation and differentiation. Recent data, however, clearly show that the ERK activation culminates in phosphorylation of many proteins with substantial regulatory functions throughout the cell, coordinating and eliciting conflicting cellular responses ranging from proliferation and differentiation to apoptosis (Peyssonnaux and Eychene, 2001; Djeu et al, 2002; Lee and McCubrey, 2002). Here, we show that in NCCIT cells, CDDP activates the MEK-ERK signalling pathway, which is followed by a caspase 3-mediated apoptosis.

\section{MATERIALS AND METHODS}

\section{Reagents}

Cisplatin was purchased from Hexal (Holzkirchen, Germany), vinblastin (vin) from Gry-Pharma (Kirchzarten, Germany) and hydrogen peroxide and two MEK1/2 inhibitors (U0126 and PD98059) from Sigma (Deisenhofen, Germany). The polyclonal antibody recognising the activated form of caspase- 3 and the inhibitor of caspase-9 (Z-LEHD-FMK) were obtained from R\&D systems (Wiesbaden, Germany). The horseradish-peroxidase (HRP)-conjugated polyclonal $\mathrm{F}(\mathrm{ab})_{2}$ fragment against digoxigenin was obtained from Dako (Hamburg, Germany), the monoclonal anti-actin antibody from Oncogene (Schwalbach, Germany), and inhibitors of caspase-3 (Ac-DEVD-CHO) and caspase-8 (Ac-IETD$\mathrm{CHO}$ ) from Pharmingen (Hamburg, Germany). The polyclonal anti-MEK1/2, anti-phospho-MEK1/2, anti-ERK1/2 and anti-phospho-ERK1/2 antibodies were purchased from Cell Signal Technology (Beverly, USA).

\section{Culturing and treatment of TGCT cell lines}

The human TGCT cell lines used in this study were NCCIT and NTERA (American Type Culture Collection, ATCC, Manassas, USA). NCCIT and NTERA cell lines were grown as monolayers and maintained in HEPES-buffered RPMI 1640 (Biochrom, Berlin, Germany) supplemented with $10 \%$ foetal calf serum (FCS) (CC Pro, Neustadt, Germany), $100 \mathrm{IU} \mathrm{ml}^{-1}$ penicillin (Sigma, Deisenhofen, Germany), $100 \mu \mathrm{g} \mathrm{ml}^{-1}$ streptomycin (Sigma) and $2 \mathrm{~mm} \mathrm{~L}-$ glutamine (Life Technologies, Karlsruhe, Germany). Cultures were incubated at $37^{\circ} \mathrm{C}$ in a humid atmosphere with $5 \%$ carbon dioxide in air. Then, they were plated subconfluent in $75 \mathrm{~mm}^{2}$ flakes and cultured in serum-free medium 1 day prior to treatment.

For the induction of apoptosis, the cells were then incubated for $2 \mathrm{~h}$ with different concentrations of $\operatorname{CDDP}(12.5,25$ and $50 \mu \mathrm{M})$, vinblastin $(15,20$ and $30 \mu \mathrm{M})$ or hydrogen peroxide $(300,600$ and $1200 \mu \mathrm{M})$. After washing, cells were incubated in culture medium for further $6,9,12$ or $24 \mathrm{~h}$.

For the induction of necrosis, the cells were heated for 15,30 or $60 \mathrm{~min}$ at $45^{\circ} \mathrm{C}$ (heat stress) or pipetted $10-15$ times (mechanical stress) and were proved to be dead by reculturing.

\section{RNA isolation and CDNA macroarray}

For cDNA macroarray, RNA was extracted $6 \mathrm{~h}$ after CDDP treatment. Total RNA was extracted from untreated and CDDPtreated NCCIT cells using 'Atlas ${ }^{\mathrm{TM}}$ Pure Total RNA Labelling Kit' (Clontech, Heidelberg, Germany). RNA integrity and quantity was assessed on an 'Agilent Bioanalyzer 2100' with a 'RNA 6000 Nano LabChip Kit' (Agilent Technologies, Waldbronn, Germany). After DNAse I digestion and addition of biotinylated oligo(dT), $\operatorname{poly}(\mathrm{A})^{+}$RNA was enriched on streptavidin-labelled magnetic beads from $100 \mu \mathrm{g}$ total RNA. RNA $(10 \mu \mathrm{g})$ templates bound to the beads were subjected to cDNA synthesis with human CDS primer mixture and $\left[\alpha-{ }^{32} \mathrm{P}\right] \mathrm{dATP}$. Purified cDNA probes $\left(>6.5 \times 10^{6}\right.$ c.p.m.) were hybridised to the arrayed human cDNA membranes (Atlas Human Apoptosis Array, Clontech) under recommended conditions. Washed membranes were exposed against the imaging plate for $12 \mathrm{~h}$ at room temperature and hybridisation signals were semiquantified on the Personal Molecular Imager FX System (BioRad, München, Germany) using an image analysis software (BioRad). Gene transcripts that displayed two-fold or greater changes were considered significant.

\section{Reverse transcription-PCR}

Total cellular RNA from pelleted cells was extracted using RNeasy Mini Kit (Qiagen, Hilden, Germany). RNA integrity and quantity was assessed on an Agilent Bioanalyser 2100 with a RNA 6000 Nano LabChip ${ }^{\mathbb{R}}$-Kit (Agilent Technologies, Waldbronn, Germany). Reverse transcription of $500 \mathrm{ng}$ total cellular RNA with random hexamer primers was performed with Omniscript RT Kit (Qiagen). Expression of MEK1 and MEK2 was quantitated on an iCycler iQ real-time detection system (BIORAD, Munich, Germany) with HotStar Taq DNA Polymerase Kit (Qiagen). The $20 \mu \mathrm{l}$ reaction from the kit was supplemented with $2 \mu \mathrm{l}$ cDNA, $0.6 \mu \mathrm{M}$ gene-specific primers and SYBR green (MoBiTec, Göttingen, Germany). Primers (MWG, Ebersberg, Germany) were designed using primer3 on-line primer design program (www-genome.wi.mit.edu/cgi-bin/primer/primer3_www.cgi).

MEK1 forward primer: 5'-TGAGAAGATCAGTGAGCTGG-3'; MEK1 reverse primer: $5^{\prime}$-ACTTGATCCAGAGAACCTCC-3'; MEK2 forward primer: 5'-AACTCAAAGACGATGACTTCG-3'; MEK2 reverse primer: $5^{\prime}$-CCATGCAAATGCTGATCTCC-3'. Acquisition of fluorescence signals was monitored on the iCycler and terminated, when all reactions reached an amplification plateau while a template-free control remained at basement level. Data analysis was carried out with iCycler iQ real-time detection system software (BioRad). To verify that only specific PCR products evoked fluorescence signals, PCR products were analysed by melt curve (BioRad iCycler) and run on $2 \%$ agarose gels for densitometry with E.A.S.Y. Win 32 software (HEROLAB, Wiesloch, Germany).

\section{Morphological detection, identification and quantification of apoptosis}

May-Giemsa-Grunwald staining Centrifuged cells $\left(2 \times 10^{3}\right)$ were dried for $24 \mathrm{~h}$, fixed in $100 \%$ acetone for $10 \mathrm{~min}$, stained with May-Giemsa-Grunwald and embedded in 'SuperMount Medium' (Dako). Apoptotic cells were identified by cellular shrinkage, nuclear condensation and fragmentation.

In situ end labelling (ISEL) Fixed centrifuged cells $\left(2 \times 10^{3}\right)$ were rinsed with TBS ( $50 \mathrm{~mm}$ Tris- $\mathrm{HCl} ; 150 \mathrm{~mm} \mathrm{NaCl} ; \mathrm{pH} 7,5)$ containing $10 \%$ FCS and $0.3 \% \mathrm{H}_{2} \mathrm{O}_{2}$ to block endogenous peroxidase activity. The cells were then incubated for $60 \mathrm{~min}$ at $37^{\circ} \mathrm{C}$ with $50 \mu \mathrm{l}$ of the labelling $\operatorname{mix}\left(250 \mathrm{U} \times \mathrm{ml}^{-1}\right.$ terminal transferase, $20 \mu \mathrm{L} \mathrm{ml}^{-1}$ digoxigen-DNA labelling $\operatorname{mix}$ at $10 \times$ concentration, and $1 \mathrm{mmoll}^{-1} \mathrm{CoCl}_{2}$ in reaction buffer for terminal transferase (Roche, Mannheim, Germany)). After being rinsing in TBS, the cells were blocked with 10\% FCS and incubated for $60 \mathrm{~min}$ with a rabbit HRP-conjugated $\mathrm{F}(\mathrm{ab})_{2}$ fragment against digoxigenin (working dilution of $1: 500 ;$ Dako). 3,3'-Diaminobenzidine (DAB) was next applied as chromogen. Cells with fragmented DNA revealed nuclear brown signals. DNA-fragmented cells with intact plasma membrane were considered to be apoptotic. Negative controls were stained as above but without terminal transferase.

Immunocytochemistry Fixed centrifuged cells $\left(2 \times 10^{3}\right)$ were rinsed in TBS containing $10 \%$ FCS and $0.3 \% \mathrm{H}_{2} \mathrm{O}_{2}$ for $10 \mathrm{~min}$. Subsequently, cells were incubated with the polyclonal antibody 
against active caspase- 3 for $30 \mathrm{~min}$. After washing in TBS, cells were further incubated with a biotin-conjugated polyclonal goat anti-rabbit antibody (Dako) for another $20 \mathrm{~min}$. The cells were then incubated with HRP-conjugated streptavidin (Dako) for 20 min. The signals were visualised with DAB. Positive cells showed a brown cytoplasmic staining around the clearly demarcated nuclei. Controls were stained as above omitting primary and secondary antibody.

Identification and quantification of apoptosis Apoptotic cells were identified by nuclear condensation and fragmentation in MGG staining, by positive cytoplasmic signals for the active form of caspase-3 in immunocytochemistry or by DNA fragmentation in ISEL. Percentage of apoptotic cells was calculated as the ratio of apoptotic cells to 500 cells counted. All experiments were repeated three times with similar results.

\section{Fluorogenic substrate assay for caspase activity}

Caspase-3, caspase- 8 and caspase- 9 activities were examined using commercially available fluoremetric kits (caspase- 3 and -8 assays, Santa Cruz Biotechnology, Heidelberg, Germany; caspase- 9 assay, BioRad). Following harvesting and centrifugation, cells $\left(1 \times 10^{5}\right)$ were lysed in $100 \mu \mathrm{l}$ lysis buffer and incubated for $10 \mathrm{~min}$ in $100 \mu \mathrm{l}$ of the reaction buffer supplemented with $100 \mu \mathrm{M}$ of the fluorogenic peptide substrate Ac-DEVD-AMC, Ac-IETD-AMC, or Ac-LEHDAMC to measure caspase- $3,-8$, or -9 activity, respectively. When necessary caspase activity was inhibited by Ac-DEVD-CHO (specific inhibitor of caspase 3), or by Ac-IETD-CHO (specific inhibitor of caspase-8) or by Z-LEHD-FMK (specific inhibitor of caspase-9) as recommended by the manufacturer. After $1 \mathrm{~h}$, the release of fluorescent 7-amino-4-trifluoromethyl coumarin was measured at 5-min intervals on a 'Fluoroskan Ascent' (Labsystems, Helsinki, Finland). All experiments were performed in triplicate and repeated three times. The data were expressed as the increase of fluorescence as a function of time.

\section{Western blot}

Tumour cells were harvested at $10^{7}$ cells in $500 \mu \mathrm{l}$ of lysis puffer ( $0.4 \%$ sodium deoxycholate, $1 \%$ NP-40, 50 mm EGTA, pH 7.4, $10 \mathrm{~mm}$ Tris $\mathrm{pH} 7.4,1 \mathrm{~mm}$ phenylsulphonyl fluoride (PMSF), $10 \mu \mathrm{g} \mathrm{ml}^{-1}$ leupeptin, $10 \mu \mathrm{g} \mathrm{ml}^{-1}$ aprotinin). After standing on ice for $20 \mathrm{~min}$, the cell lysates were cleared by centrifugation at $5000 \mathrm{~g}$ for $10 \mathrm{~min}$. The cytosolic supernatants containing equal amounts of protein were then resolved by electrophoresis on SDS - PAGE gel $\left(10 \%\left(\mathrm{w} \mathrm{v}^{-1}\right)\right.$ gel). Thereafter, the proteins were transferred electrophoretically to a nitrocellulose membrane (Schuett, Goettingen, Germany). After blocking in $10 \mathrm{~mm}$ Tris-HCl buffer, $\mathrm{pH}$ 7.4, containing $150 \mathrm{~mm} \mathrm{NaCl}, 0.1 \%$ Tween 20 and $5 \%\left(\mathrm{wv}^{-1}\right)$ nonfat dry milk, the membrane was treated with appropriate primary antibodies followed by incubation with HRP-conjugated secondary antibodies. The antigen - antibody complexes were detected using a chemiluminescence reagent kit (Amersham Pharmacia Biotech, Freiburg, Germany).

\section{Cell cycle analysis}

To exclude that MEK inhibitors impair the cell cycle, NCCIT cells were stained by CycleTEST ${ }^{\mathrm{TM}}$ Plus DNA Reagent Kit (Becton Dickinson, Heidelberg, Germany) and analysed by flow cytometry (FACSCalibur, Becton Dickinson), as described by others (Bach et al, 1991).

\section{RESULTS}

\section{Dose- and time-dependent effects of CDDP}

Dose-dependent effects of CDDP were determined $24 \mathrm{~h}$ after application of $12.5,25$ or $50 \mu \mathrm{M}$ CDDP to the NCCIT cells. To assess the apoptotic rate in addition to MGG, we applied two other morphological methods, namely ISEL for DNA fragmentation and immunocytochemistry for the active form of caspase 3. A concentration of $50 \mu \mathrm{M}$ CDDP was defined as the optimal drug
A
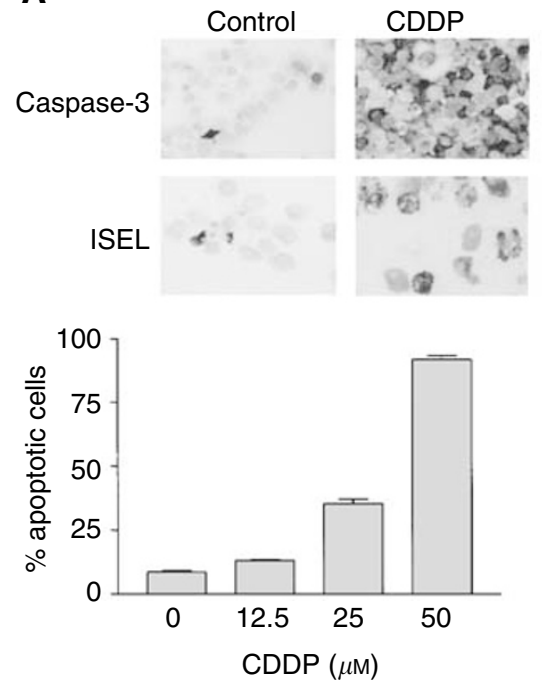

B
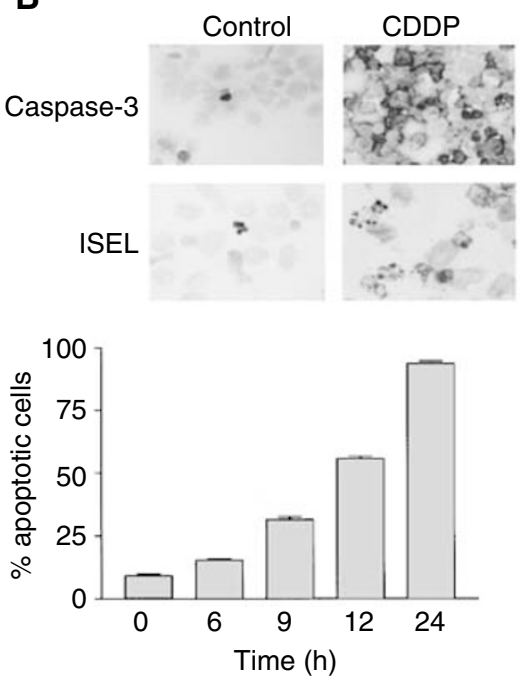

Figure I Dose- and time-dependent effects of CDDP on NCCIT cells. (A) Tumour cells were treated with different CDDP concentrations. After $24 \mathrm{~h}$, the apoptotic rate of tumour cells was assessed by MGG staining, immunocytochemistry for the active form of caspase-3 and ISEL for DNA fragmentation. Representative photomicrographs of active caspase-3 (black cytoplasmatic signals) and of DNA fragmentation (black nuclear signals) in untreated (control) and drug-treated NCCIT cells illustrate increased apoptosis of tumour cells treated with $50 \mu \mathrm{M}$ CDDP when compared to the control. The diagram shows quantification of tumour cell apoptosis with MGG staining following application of different CDDP concentrations. (B) Tumour cells were treated with $50 \mu \mathrm{M}$ CDDP. After 6, 9, 12 and 24h, the apoptotic rate of tumour cells was assessed. Representative photomicrographs of active caspase-3 and of DNA fragmentation in untreated and CDDP-treated NCCIT cells illustrate increased apoptosis of tumour cells $24 \mathrm{~h}$ after drug treatment. The diagram shows quantification of tumour cell apoptosis with MGG staining in time dependency. Data presented in $(\mathbf{A})$ and $(\mathbf{B})$ are the mean of triplicates; similar results were obtained in three separate experiments. 
concentration to elicit apoptosis in $93 \pm 5 \%$ of tumour cells within $24 \mathrm{~h}$ (Figure 1). Experiments with this optimal CDDP concentration revealed that the apoptotic rate of NCCIT cells increased from $9 \pm 2 \%$ at the beginning to $15 \pm 1 \%$ at $6 \mathrm{~h}, 31 \pm 2 \%$ at $9 \mathrm{~h}, 54 \pm 4 \%$ at $12 \mathrm{~h}$ and $93 \pm 5 \%$ at $24 \mathrm{~h}$ after drug application (Figure 1).

\section{Analysis of apoptosis-regulating genes following CDDP treatment}

On the strength of the evidence that the apoptotic rate of tumour cells showed an initial increase at $6 \mathrm{~h}$ after drug treatment, we performed a cDNA macroarray analysis to compare the expression pattern of 205 apoptosis-related genes in NCCIT cells before and $6 \mathrm{~h}$ after application of $50 \mu \mathrm{m}$ CDDP. Most of the genes analysed, however, were either not detectable (no signal) or only weakly expressed in NCCIT cells (weak signals) and did not reveal any down- or upregulation after drug treatment. p53, the p53-regulated gene $\mathrm{mdm}-2$ and genes from the $\mathrm{Bcl}-2$ family belonged to this group, as reported by others (Burger et al, 1997; Kersemaekers et al, 2002) (Table 1). The expression level of five genes including IGFBP-2, AP-1, CDC25A, GADD45 and ID-1 was decreased in treated cells compared to the levels in untreated cells (Table 1). In contrast, the expression of 12 genes including those involved in cell cycle and apoptosis (e.g. CDK4, CDK5, cyclin B1 and caspase3 ), or in signal transductions (e.g. GRB2 and PLK1) was increased in CDDP-treated cells when compared to untreated ones (Table 1). The fourth category of genes included those with a sustained high level of expression before and after drug treatment. MEK1/2, RHOA, Prefoldin and N-myc belonged to this category of genes (Table 1).

Owing to the high and sustained expression level of MEK1/2 mRNA, and the fact that activation of the MEK-ERK pathway cannot only hamper but also promote the sensitivity of tumour cells to CDDP (Persons et al, 1999; Wang et al, 2000), in the followup this study was focused on the role of MEK in the course of drug-induced apoptosis. At first, we proved the expression of MEK1/2 mRNA by a second method. Semiquantitative RT - PCR confirmed the results from the macroarray and revealed that in NCCIT cells the transcription level of both MEK1 and MEK2

Table I Expression of apoptosis-related genes in NCCIT cells following CDDP treatment

\begin{tabular}{lll}
\hline CDK4 & Cyclin-dependent kinase 2 & Increased \\
CDK5 & Cyclin-dependent kinase 4 & Increased \\
Cyclin BI & Cyclin B & Increased \\
Caspase-3 & Caspase-3 & Increased \\
GRB-2 & Growth factor receptor-bound protein 2 & Increased \\
PLK & Polo-like kinase & Increased \\
p53 & p53 tumor suppressor protein & Unchanged \\
mdm-2 & p53-binding mouse double minute 2 homolog & Unchanged \\
bcl-2 & B-cell leukemia/lymphoma protein 2 & Unchanged \\
bax & Bcl-2-associated X protein membrane & Unchanged \\
IGFBP-2 & IGF-binding protein 2 & Decreased \\
AP-1 & Transcription factor & Decreased \\
CDC25A & Cell division cycle 25 homolog A & Decreased \\
GADD45 & Growth arrest and DNA damage-inducible & Decreased \\
& protein 45 & \\
ID-I & Inhibitor of DNA-binding protein I & Decreased \\
MEKI/2 & Mitogen-activated protein kinase kinase I/2 & Sustained high \\
RHOA & Ras homolog gene family member & Sustained high \\
Prefoldin 5 & c-myc-binding protein & Sustained high \\
N-myc & N-myc & Sustained high \\
\hline
\end{tabular}

Two RNA preparations were isolated from untreated and CDDP-treated NCCIT cells. RNAs were reverse transcribed, and generated cDNA probes were hybridised to cDNA macroarray membrane filters. Gene transcripts that displayed two-fold or greater changes were considered significant. Only 17 differentially expressed genes were identified among 205 genes investigated.
mRNAs remained constant after drug treatment (Table 1, Figure 2). As control, we investigated the expression level of MEK1/2 mRNA after heat- and mechanically induced necrosis of the NCCIT cell line. Results showed that stress stimuli leading to cell death without primary DNA damage (heat- or mechanically induced necrosis) do not elicit any significant change in the expression level of MEK1/2 mRNA. Figure 2 illustrates the expression level of MEK1 and MEK2 mRNAs following heat-induced necrosis in NCCIT cells.

\section{CDDP-induced activation of the MEK-ERK signalling pathway}

Next, lysates from untreated and drug-treated tumour cells were analysed by Western blot for the expression of MEK1/2 and its substrate ERK1/2. The same blots were stripped and analysed with antibodies recognising dually phosphorylated MEK (pMEK) $1 / 2$ or dually phosphorylated ERK (pERK) $1 / 2$ to verify the active fraction of each protein in our experiments. Results illustrated that, despite high expression, MEK1/2 and ERK1/2 proteins were mainly unphosphorylated in untreated NCCIT cells (Figure 3). However, phosphorylation of $\mathrm{MEK} 1 / 2$ and ERK1/2 proteins began to increase at $6 \mathrm{~h}$ and was at its highest at $12 \mathrm{~h}$ after CDDP treatment, a course which has been observed in growth factor- or oxidantmediated MEK-ERK activation in many cell types (Jimenez et al, 1997; Wang et al, 1998). Interestingly, MEK1 and ERK2 seem to be more strongly phosphorylated than MEK2 and ERK1.

\section{Blocking of CDDP-induced apoptosis by MEK inhibitors}

To study whether ERK activation is required for CDDP-induced apoptosis in NCCIT cells, two chemical MEK1/2 inhibitors, U0126 and PD98059, were used. Cells were treated with various doses of MEK1/2 inhibitors simultaneously to the addition of $50 \mu \mathrm{M}$ CDDP. Both inhibitors reduced the grade of ERK phosphorylation (Figure 4). Morphological quantification of apoptotic cells by MGG, immunostaining for active caspase 3 and ISEL for fragmented DNA demonstrated that application of an inhibitor significantly reduced the CDDP-induced apoptosis of tumour cells in a dose-dependent manner when compared to controls. For example, application of $30 \mu \mathrm{M}$ U0126 was capable of decreasing the rate of apoptosis of drug-treated cells from approximately 95 to about $50 \%$. Results are shown in Figure 4. To rule out that MEK inhibitors unspecifically influence the cell cycle and thereby interfere with apoptosis, flow cytometric DNA analysis of NCCIT cells were carried out 6 and $24 \mathrm{~h}$ after treatment with U0126 or PD98059 $(50 \mu \mathrm{M})$. Results showed that MEK inhibitors do not impair the cell cycle of NCCIT cells (Figure 4).

\section{MEK-ERK activation results in caspase activity}

By applying caspase assays, we first analysed the activation of initiating caspase- 8 and -9 and of the executing caspase- 3 in the course of CDDP-induced apoptosis in NCCIT cells. Results demonstrated that the caspase-8 and -9 activity increased 3-4fold, and the activity of caspase- 3 about $14-16$-fold at $24 \mathrm{~h}$ after CDDP application (Figure 5). Next we investigated the activity of caspase-8, -9 and -3 following MEK inhibition to ascertain that the increased activation of these caspases depended on the MEK-ERK activation. Indeed, application of MEK inhibitors (U0126 or PD98059) substantially decreased the activity of all three caspases (Figure 5). We studied then the biological influence of each caspase for apoptosis on drug-treated NCCIT cells. We noted that $24 \mathrm{~h}$ after CDDP application, the apoptotic rate of tumour cells decreased from $93 \pm 5$ to $75 \pm 4 \%$ following inhibition of caspase- 8 , to $85 \pm 3 \%$ following inhibition of caspase- 9 , or to $49 \pm 6 \%$ following inhibition of caspase-3 (Figure 5). Moreover, we observed that the activity of caspase- 3 was only weakly reduced when caspase- 8 or -9 
A

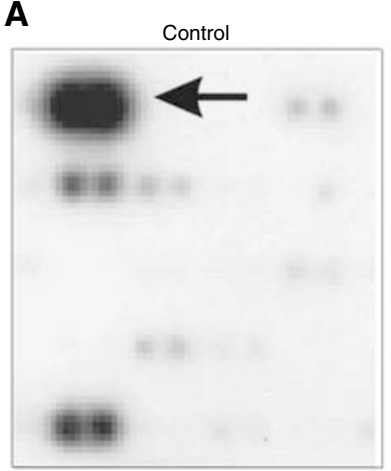

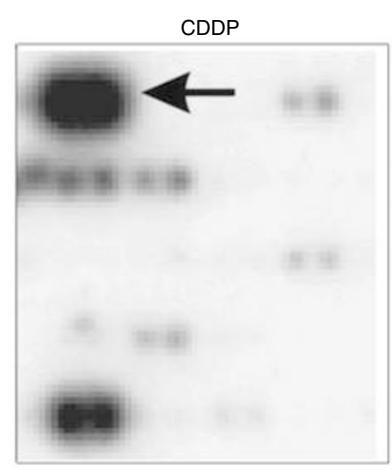

B
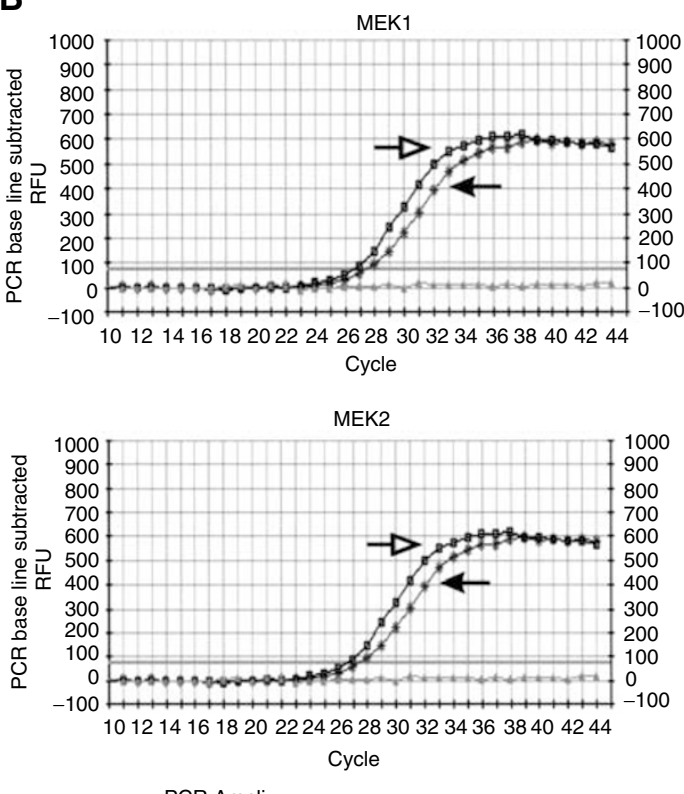

PCR Ampli

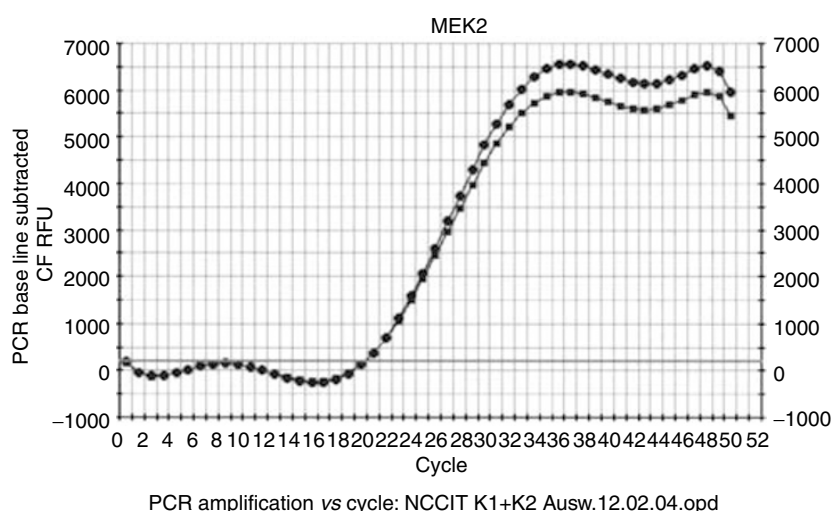

C

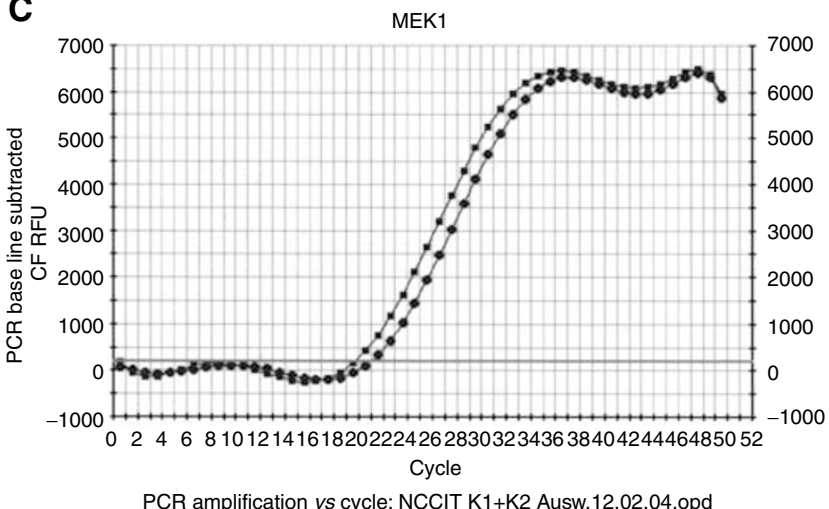

Figure 2 MEKI/2 mRNA expression in untreated and CDDP-treated NCCIT cells. (A) Two RNA preparations from untreated and CDDP-treated
NCCIT cells were reverse transcribed and hybridised to cDNA macroarray membrane filters. Sections from two representative autoradiographies show sustained expression of MEKI/2 in untreated (control) and CDDP-treated $(50 \mu \mathrm{M})$ NCCIT cells $6 \mathrm{~h}$ after drug treatment (arrows). (B) semiquantitative realtime RT-PCR of MEKI and MEK2 mRNA confirms that the expression level of both transcripts remains constant in CDDP-treated NCCIT cells (open arrows, left) compared to untreated ones (closed arrows, right). (C) as control, the expression level of MEKI and MEK2 mRNA after heat-induced necrosis of the NCCIT cell line was analysed. Results demonstrate that in the course of heat-induced necrosis, the expression level of MEK mRNAs is not changed (-) compared to untreated ones (-

was inhibited (Figure 5). Thus, caspase-8 and -9 seem to play only a supporting role, whereas caspase- 3 is the main caspase responsible for the CDDP-induced apoptosis in NCCIT cells.

\section{Selectivity of CDDP-induced apoptosis}

To prove whether activation of the MEK/ERK pathway seen in the p53-mutated TGCT cell line NCCIT is also required for the CDDPinduced apoptosis in human neoplastic germ cells with wt-p53, NTERA cells were treated with CDDP. As reported by others (Chresta et al, 1996; Burger et al, 1999), CDDP treatment led to apoptosis of NTERA cells dose and time dependently (data not shown). Western analysis showed that following CDDP application $(50 \mu \mathrm{M})$ both MEK and ERK were phosphorylated and simultaneous treatment of NTERA cells with CDDP and one of the MEK1/ 2 inhibitors U0126 $(30 \mu \mathrm{M})$ or PD98059 $(50 \mu \mathrm{M})$ significantly reduced the apoptosis. Notably, the effect of MEK1/2 inhibitors on the apoptotic rate of drug-treated NTERA cells was as efficient as that seen in NCCIT cells (Figure 6). Thus, we concluded that the CDDP-induced apoptosis of NCCIT and NTERA cells was, at least partially, p53 independent and mediated by the MEK/ERK signalling pathway.

\section{Specificity of CDDP-induced apoptosis}

To examine the role of the MEK-ERK signalling pathway in the induction of apoptosis and/or necrosis following other stress stimuli, NCCIT cells were treated with various doses of vinblastin $(15-30 \mu \mathrm{M})$ - as a relevant drug in the therapy of TGCT - and of hydrogen peroxide $\left(\mathrm{H}_{2} \mathrm{O}_{2} ; 300-1200 \mu \mathrm{M}\right)$ or underwent heat $\left(45^{\circ} \mathrm{C}\right.$ up to $60 \mathrm{~min}$ ) or mechanical stress (multiple pipetting). Figure 7 demonstrates that treatment with vinblastin and $\mathrm{H}_{2} \mathrm{O}_{2}$ led to apoptosis of NCCIT cells in a dose-dependent manner and was conducted by ERK activation. As expected, MEK inhibition led to a significant reduction in apoptosis in NCCIT cells and was as effective as that seen in experiments with CDDP and MEK1/2 


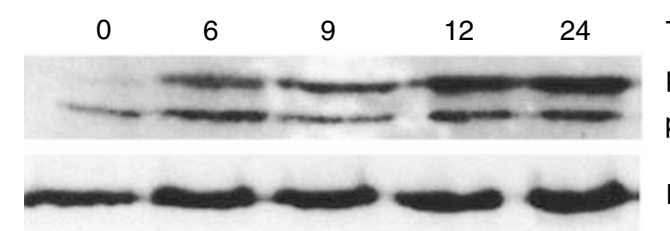

Time $(\mathrm{h})$

PMEK 1

PMEK 2

MEK $1 / 2$

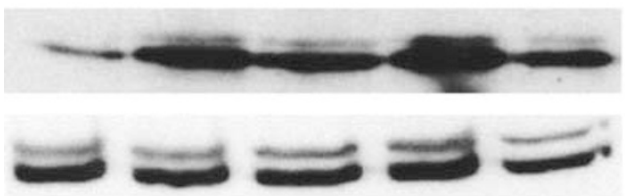

pERK 1

pERK 2

ERK $1 / 2$

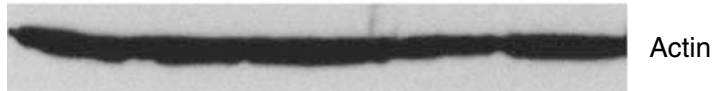

Figure 3 CDDP-induced activation of the MEK/ERK pathway in NCCIT cells. NCCIT cells were treated with $50 \mu \mathrm{M}$ CDDP for $2 \mathrm{~h}$, after which cell lysates were investigated for the expression and activation (phosphorylation) of MEKI/2 and ERKI/2 by Western blot analysis in the time course. Upper panel illustrates the expression of MEKI/2 and its activation (phosphorylation) following CDDP treatment. Lower panel demonstrates results from Western blots of ERKI/2 and its activated (phosphorylated) form pERKI/2 in the time course after CDDP treatment. Note that MEKI and ERK2 are more strongly phosphorylated than MEK2 and ERKI. Expression of actin was used to control equal protein loading.

inhibitors U0126 or PD98059 (data not shown). Results also show that stress stimuli leading to cell death without primary DNA damage (heat- or mechanically induced necrosis) elicit ERK phosphorylation (Figure 7).

\section{DISCUSSION}

The present study was focused on p53-independent mechanisms involved in CDDP-induced apoptosis of the human neoplastic germ cell line NCCIT, the p53 protein of which is known to remain inactive during the course of drug-induced apoptosis (Burger et al, 1997; Burger et al, 1999). To address this issue, we first studied dose- and time-dependent effects of CDDP on NCCIT cells. Experiments indicated that following application of an optimal concentration of $50 \mu \mathrm{M}$ CDDP, the apoptotic rate of tumour cells increased from approximately $10 \%$ at the beginning to about $95 \%$ at $24 \mathrm{~h}$. Significant differences in the degree of apoptosis between CDDP-treated and untreated cells were, however, not observed before $6 \mathrm{~h}$. In the light of previous data indicating that CDDP exerts its cytotoxicity via the formation of mono-, inter-, and intrastrand CDDP-DNA adducts, which can ultimately result in cell cycle arrest at G1, S or G2-M and in the induction of apoptosis (Fichtinger-Schepman et al, 1985; Sorenson and Eastman, 1988; Eastman, 1990), we hypothesised that within $6 \mathrm{~h}$ after application CDDP upregulated proapoptotic and/or downregulated antiapoptotic genes, thus mediating apoptosis in NCCIT cells. For a scanning view of differentially regulated genes, we investigated the expression profile of 205 apoptosis-related genes by applying a commercially available cDNA macroarray. As expected, no significant changes were found in the expression levels of p53 and the p53-dependent gene mdm-2 upon drug application. In addition to a few differentially regulated genes with unknown function for the course of CDDP-induced apoptosis, we noted a sustained high level of MEK1/2 mRNA expression in NCCIT cells before and after drug treatment.

MEK1 and MEK2 are protein kinases that when activated (phosphorylated) are believed to dually phosphorylate only ERK1
A

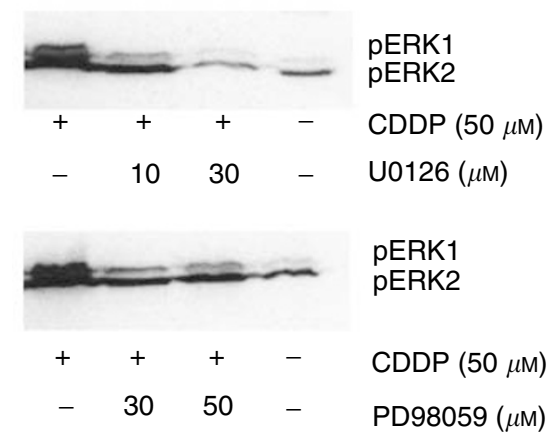

B

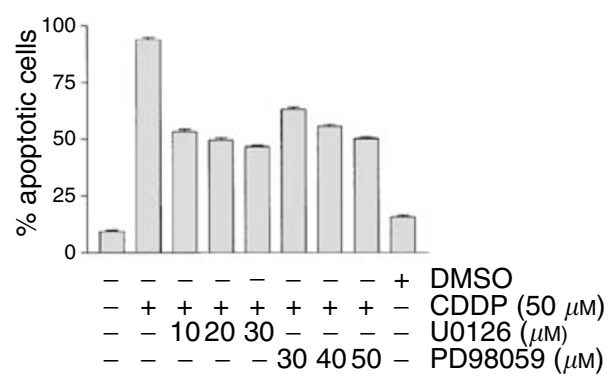

C

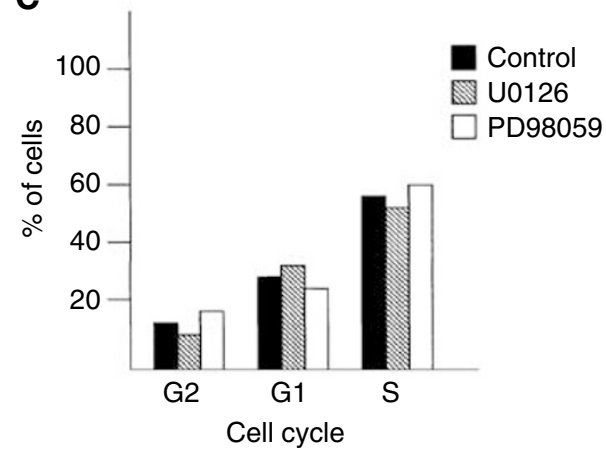

Figure 4 Blocking of CDDP-induced apoptosis by MEK inhibitors. To investigate whether phosphorylation of ERKI/2 is required for the CDDPinduced apoptosis of NCCIT cells, two different MEK inhibitors, UOI 26 and PD98059, were applied in addition to $50 \mu \mathrm{M}$ CDDP. (A) Western blot analysis with an anti-phospho-ERKI/2 antibody shows the inhibition of ERKI/2 activation by MEKI/2 inhibitors ( $U 0$ I 26, 10 and $30 \mu \mathrm{M}$; PD98059, 30 and $50 \mu \mathrm{M}$ ). (B) The diagram shows quantification of tumour cell apoptosis with MGG staining dependent on ERK blockage by different concentration of MEK inhibitors after $24 \mathrm{~h}$. Data are the mean of triplicates; similar results were obtained in three separate experiments. (C) Flow cytometric DNA analysis of NCCIT cells treated with the MEK inhibitor U0I26 $(50 \mu \mathrm{M})$ demonstrates that following drug treatment, the cell cycle of NCCIT cells is not changed.

and ERK2, thereby increasing the enzymatic activity of ERKs approximately 1000 -fold over the activity found with the basal or monophosphorylated forms (Traverse et al, 1992; Lenormand et al, 1993, Robbins et al, 1993). The consequence is a phosphorylation of diverse protein kinases, transcription factors, and even cytoskeletal proteins leading to paradoxical cellular responses ranging from proliferation to apoptosis (Peyssonnaux and Eychene, 2001; Djeu et al, 2002; Lee and McCubrey, 2002). Interestingly, similar conflicting reports also exist on the role of the MEKs and ERKs in CDDP-induced apoptosis. For example, the 
A
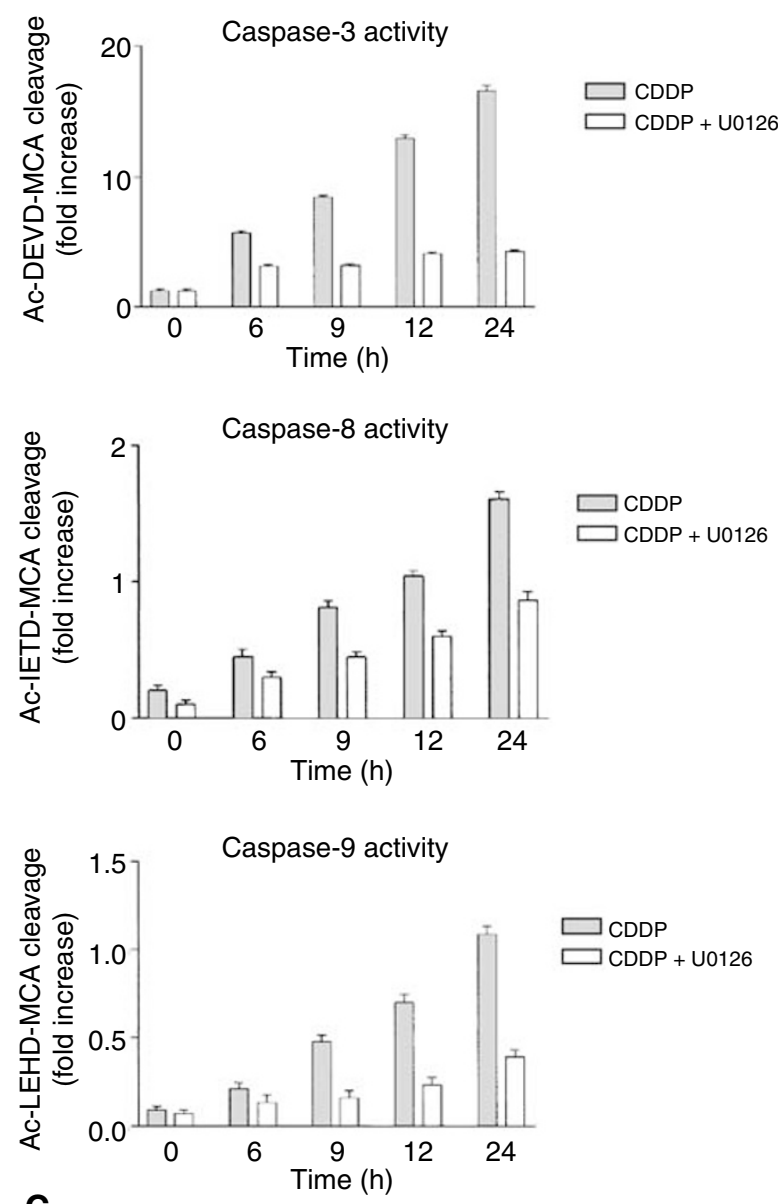

政

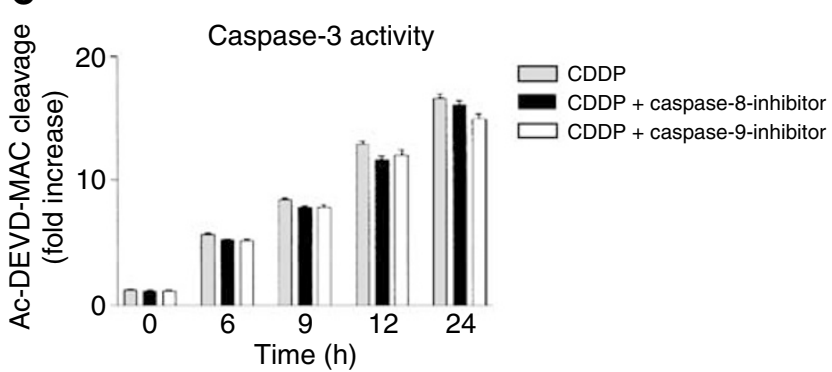

\section{B}
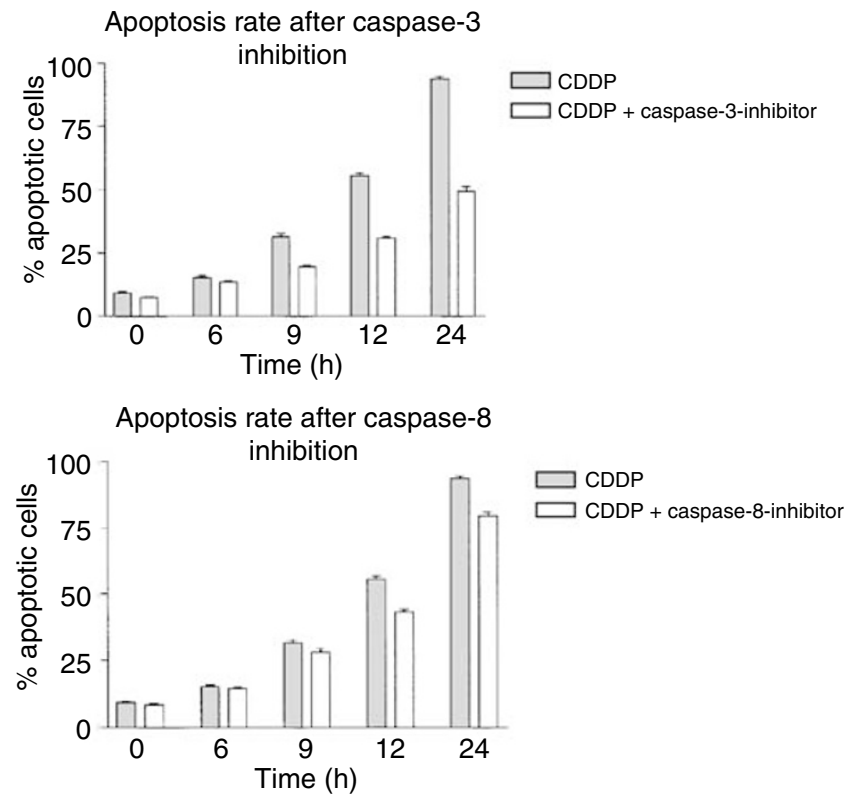

Apoptosis rate after caspase-9

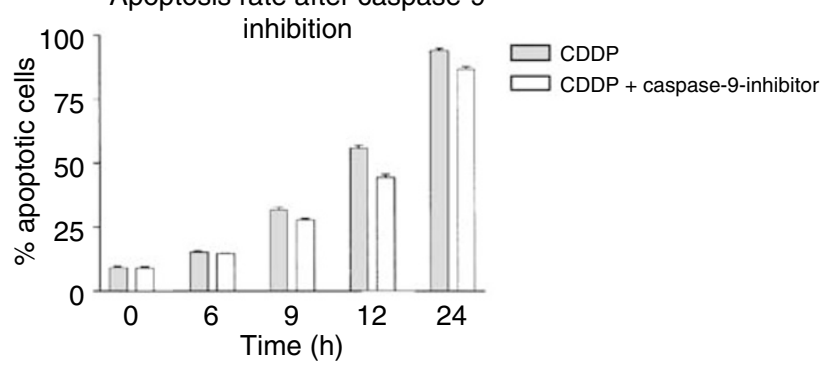

Figure 5 MEK-ERK activation results in caspase activity. (A) NCCIT cells were treated with CDDP (50 $\mu \mathrm{M})$ in the presence or absence of the MEK inhibitor $\cup 0 \mathrm{I} 26(30 \mu \mathrm{M})$ as described in Materials and methods. After cell lysis, the activity of caspase-3, -8 and -9 was determined using specific fluorogenic substrates: Ac-DEVD-MCA (for caspase-3). Ac-IETD-MCA (for caspase-8) and Ac-LEHD-MCA (for caspase-9). The data are expressed as the increase in fluorescence as a function of time. Note that the $Y$-axis ranges from 0 to 20 for caspase-3, from 0 to 2 for caspase- 8 and from 0 to I.5 for caspase-9. (B) $\mathrm{NCCIT}$ cells were treated with CDDP $(50 \mu \mathrm{M})$ in the presence or absence of Ac-DEVD-CHO as caspase-3 inhibitor, or Ac-IETD-CHO as caspase-8 inhibitor or Z-LEHD-FMK as caspase-9 inhibitor. Diagrams show quantification of tumour cell apoptosis with MGG staining in time dependency. (C) NCCIT cells were treated with CDDP $(50 \mu \mathrm{M})$ in the presence or absence of Ac-IETD-CHO as caspase-8 inhibitor or Z-LEHD-FMK as caspase-9 inhibitor. After cell lysis, the activity of caspase-3 was determined. Results show the increase in fluorescence as a function of time. Data presented in (A-C) are the mean of triplicates; similar results were obtained in three separate experiments.

activity of the MEK-ERK signalling pathway seems to reduce sensitivity to CDDP in ovarian carcinoma cells, whereas phosphorylation of MEK and ERK has been found to be essential for the CDDP-induced apoptosis in cervical carcinoma cells (Persons et al, 1999; Wang et al, 2000).

To prove the results from the cDNA macroarray analysis and to address the role of the MEK-ERK signalling pathway in neoplastic germ cells, semiquantitative RT-PCR and immunoblots were carried out. The findings from the real-time RT-PCR were in agreement with those from the cDNA macroarray analysis and indicated that up to $24 \mathrm{~h}$ after CDDP application the expression level of MEK1 and MEK2 mRNAs remained constant. Immunoblots supported these data and demonstrated that both MEK1/2 and ERK1/2 proteins were strongly expressed but were mainly unphosphorylated and thereby inactive in untreated tumour cells. Interestingly, CDDP treatment resulted in high and sustained 
A

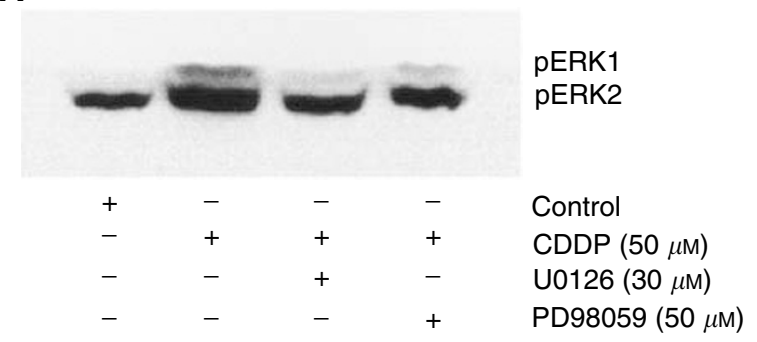

B

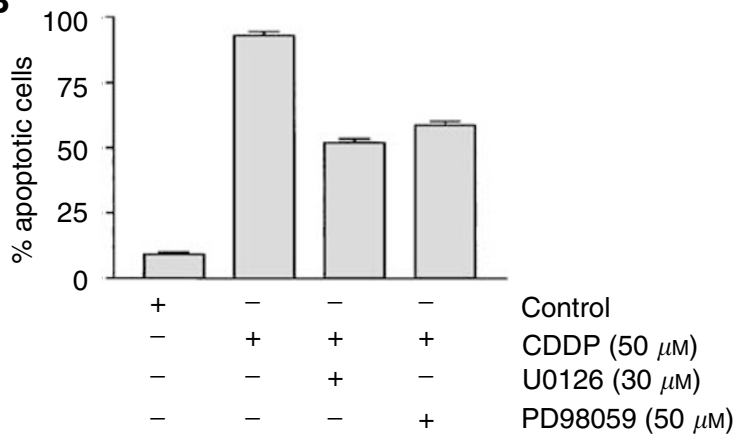

Figure 6 Selectivity of CDDP-induced apoptosis. NTERA cells were treated with $50 \mu \mathrm{M}$ CDDP in the presence or absence of the MEK inhibitors U0 I $26(30 \mu \mathrm{M})$ or PD98059 (50 $\mu \mathrm{M})$, as described in Material and methods. (A) After $24 \mathrm{~h}$, tumour cells were lysed and investigated for the expression and activation (phosphorylation) of ERKI/2 by Western blot analysis. (B) the diagram shows quantification of tumour cell apoptosis with MGG staining dependent on ERK blockage by MEK inhibitors after $24 \mathrm{~h}$. Data are the mean of triplicates; similar results were obtained in three separate experiments. activation of MEK, particularly MEK1, and ERK, especially ERK2, which was strongly correlated to the apoptosis of tumour cells. Thus, it is tempting to speculate that a prolonged period of excessive phosphorylation of ERK may be necessary for its biological effect on TGCT as demonstrated for other cells types (Traverse et al, 1992; Lenormand et al, 1993).

To clarify the importance of the MEK-ERK pathway for the apoptosis of drug-treated NCCIT cells, we interrupted the pathway activity by using two different MEK inhibitors. Experiments revealed that the MEK inhibitors not only downregulated the level of ERK phosphorylation but also reduced the apoptotic rate of CDDP-treated NCCIT cells, as previously reported for the cervical carcinoma cell line HeLa (Wang et al, 2000). Results from RT PCR and Western analyses, however, suggested that the CDDPinduced apoptosis in NCCIT cells depended on phosphorylation of MEK and ERK constitutively expressed in tumour cells and did not require any MEK/ERK de novo expression.

Considering the pivotal role of caspases in the initiation and execution of apoptosis (Thornberry and Lazebnik, 1998), we next analysed the activation of caspase- $3,-8$ and -9 following CDDP treatment of NCCIT cells. Caspases are divided into two subfamilies, initiator (e.g. caspase-8) and executor (e.g. caspase3 ), based on their roles in the apoptotic signalling cascade (Salvesen and Dixit, 1997; Nunez et al, 1998). Depending on apoptotic signal and cell type, activation of initiator caspases may lead to mitochondria-dependent and/or -independent activation of executor caspases; whereas the former requires cytochrome $c$ release and activation of caspase- 9 as consequences of mitochondria damage, the latter is characterised by a cytochrome- $c$ - and caspase-9-independent activation of executor caspases (Krammer, 2000). In this study, elevated caspase activity after CDDP application was found in caspase-3, -8 and -9 . However, caspase3 activation seemed to be more important for the course of
A
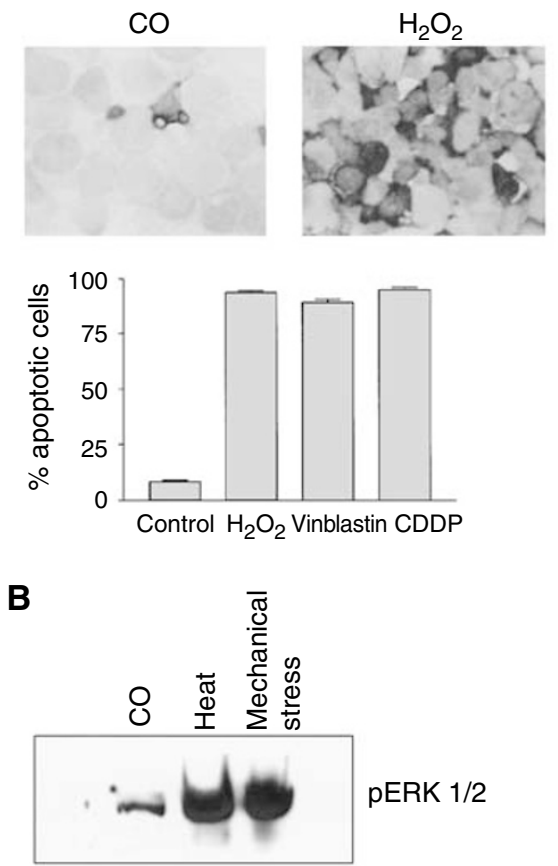
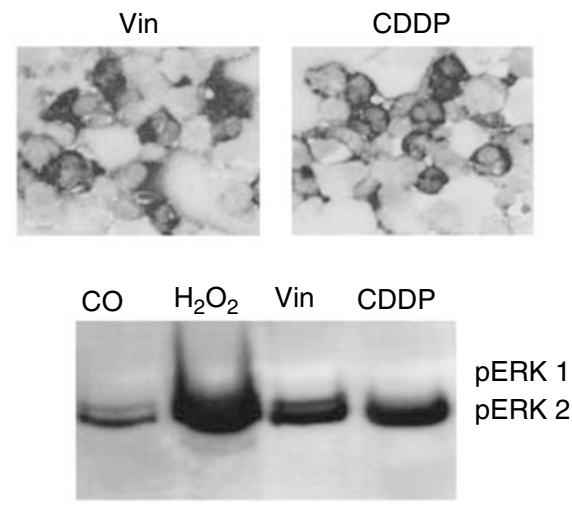

Figure 7 Specificity of CDDP-induced apoptosis. (A) NCCIT cells were treated with CDDP $(50 \mu \mathrm{M})$, vinblastin $(30 \mu \mathrm{M})$ or $\mathrm{H}_{2} \mathrm{O}_{2}($ ( $200 \mu \mathrm{M})$. After $24 \mathrm{~h}$, the apoptotic rate of tumour cells was assessed by immunocytochemistry for the active form of caspase-3 and MGG staining. Representative photomicrographs of caspase-3 (black cytoplasmatic signals) in NCCIT cells illustrate increased caspase activity in tumour cells following treatment. The diagram shows quantification of tumour cell apoptosis with MGG staining. Data are the mean of triplicates; similar results were obtained in three separate experiments. A fraction of tumour cells was lysed and investigated for the expression and activation (phosphorylation) of ERKI/2 by Western blot analysis. (B) To examine the role of the MEK-ERK signalling pathway in the course of necrosis, NCCIT cells underwent heat ( $45^{\circ} \mathrm{C}$ up to 60 min) or mechanical stress (multiple pipetting). Results demonstrate that stress stimuli leading to cell death without primary DNA damage (heat- or mechanically induced necrosis) also elicit ERK phosphorylation. 
apoptosis than caspase- 8 and -9 activation, because blockade of caspase- 3 inhibited apoptosis to a greater extent than the blockade of caspase- 8 or -9 . Consistently, the activity of caspase- 3 was only moderately abolished when caspase- 8 or -9 was blocked. Taking the published data on the involvement of caspase- 8 and -9 in mitochondria-dependent apoptosis into account (Krammer, 2000), we consider the possibility that ERK-mediated apoptosis in NCCIT cells is mainly induced by a mitochondria-independent activation of caspase- 3 .

We investigated then whether the ERK-mediated apoptosis is specific for NCCIT, and whether other apoptosis-inducing agents also activate ERK in NCCIT cells. Experiments on the human malignant germ cell line NTERA possessing the wild-type p53 had indicated that NTERA cells were as sensitive to CDDP as NCCIT cells, and apoptosis of NTERA cells was also mediated by ERK. Consequently, when MEK was blocked by specific inhibitors, the apoptotic rate of treated NTERA cells was reduced significantly. Thus, activation of the MEK-ERK pathway leads to apoptosis independent from the p53 status. Importantly, experiments with another relevant chemotherapeutic drug (vinblastin) and reactive oxygen species $\left(\mathrm{H}_{2} \mathrm{O}_{2}\right)$ revealed that following treatment of neoplastic germ cells ERK was phosphorylated, and tumour cells underwent apoptosis. Thus, activation of the MEK-ERK pathway enhances apoptosis not only in NCCIT but also in NTERA cells and the ERK-mediated apoptosis in NCCIT cells is not limited to CDDP. To answer the question whether ERK activation can also be induced by nonapoptotic stimuli inducing cell death without primary DNA damage, NCCIT cells underwent heat or mechanical stress. Results showed that these stress stimuli also led to the ERK phosphorylation. In conclusion, phosphorylation of ERK is not specific for CDDP, because other apoptosis (e.g. Vinblastin)- and necrosis-inducing stimuli (e.g. heat) are also capable to activate the ERK signalling pathway.

The question is, however, how does the MEK-ERK pathway becomes active following treatment with drugs (e.g. CDDP or vinblastin) or reactive oxygen species (e.g. $\mathrm{H}_{2} \mathrm{O}_{2}$ ). In this respect, recent data support the hypothesis that toxic substances such as reactive oxygen species may inhibit phosphatases (e.g. protein phosphatase or protein tyrosine phosphatases) and thereby contribute to the activation of ERK (Lee and Esselman, 2002) Another scenario may be that CDDP ligates growth factor receptors, thereby activating the MEK-ERK pathway, as suggested for the cervical carcinoma cell line HeLa (Wang et al, 2000). Indeed there are even some reports demonstrating that TGCT express an aberrant platelet-derived growth factor $\alpha$ receptor, which is hypothetically capable of activating the MEKERK pathway upon ligation (Kollmannsberger et al, 2002; Palumbo et al, 2002).

Other remaining questions are whether other kinases (e.g. JNK or p38) collaborate with MEK-ERK in apoptosis of malignant germ cells, as shown for some tumours (Boldt et al, 2002), and more importantly - which downstream effectors are involved in MEK-ERK-mediated apoptosis?

In conclusion, the data suggest that CDDP mediates its p53independent apoptosis-inducing effect on the malignant human testicular germ cells through activation of the MEK-ERK signalling pathway that culminates in activation of the executor caspase- 3 and thereby in programmed cell death. The data would also suggest that an antitumour therapy with CDDP should not be combined with MEK inhibitors because such combination might be antagonistic and not of benefit for the outcome of patients with TGCT.

\section{REFERENCES}

Bach BA, Knape WA, Edinger MG, Tubbs RR (1991) Improved sensitivity and resolution in the flow cytometric DNA analysis of human solid tumor specimens. Use of in vitro fine-needle aspiration and uniform staining reagents. Am J Clin Pathol 96: 615-627

Boldt S, Widle UH, Kolch W (2002) The role of MAPK pathways in the action of chemotherapeutic drugs. Carcinogenesis 23: $1831-1838$

Burger H, Nooter K, Boersma AW, Kortland CJ, Stoter G (1997) Lack of correlation between cisplatin-induced apoptosis, p53 status and expression of Bcl-2 family proteins in testicular germ cell tumour cell lines. Int J Cancer 73: $592-599$

Burger $\mathrm{H}$, Nooter K, Boersma AW, van Wingerden KE, Looijenga LH, Jochemsen AG, Stoter G (1999) Distinct p53-independent apoptotic cell death signalling pathways in testicular germ cell tumour cell lines. Int J Cancer 81: $620-628$

Chresta CM, Masters JRW, Hickman JA (1996) Hypersensitivity of human testicular tumors to etoposide-induced apoptosis is associated with functional p53 and a high Bax: Bcl-2 ratio. Cancer Res 56: $1834-1841$

Djeu JY, Jiang K, Wei S (2002) A view to a kill: signals triggering cytotoxicity. Clin Cancer Res 8: 636-640

Eastman A (1990) Activation of programmed cell death by anticancer agents: cisplatin as a model system. Cancer Cells 2: 275-280

Einhorn LH (2002) Curing metastatic testicular cancer. Proc Natl Acad Sci USA 99: $4592-4595$

Fichtinger-Schepman AM, van der Veer JL, den Hartog JH, Lohman PH, Reedijk J (1985) Adducts of the antitumor drug cis-diamminedichloroplatinum(II) with DNA: formation, identification, and quantitation. Biochemistry 24: $707-713$

Higby DJ, Wallace Jr HJ, Albert DJ, Holland JF (1974) Diaminodichloroplatinum: a phase I study showing responses in testicular and other tumors. Cancer 33: 1219-1225

Jimenez LA, Zanella C, Fung H, Janssen YM, Vacek P, Charland C, Goldberg J, Mossman BT (1997) Role of extracellular signal-regulated protein kinases in apoptosis by asbestos and $\mathrm{H}_{2} \mathrm{O}_{2}$. Am J Physiol 273: L1029 - L1035
Kersemaekers AM, Mayer F, Molier M, van Weeren PC, Oosterhuis JW, Bokemeyer C, Looijenga LHJ (2002) Role of P53 and MDM2 in treatment response of human germ cell tumors. I Clin Oncol 20: $1551-1561$

Kollmannsberger C, Mayer F, Pressler H, Koch S, Kanz L, Oosterhuis JW, Looijenga LH, Bokemeyer C (2002) Absence of c-KIT and members of the epidermal growth factor receptor family in refractory germ cell cancer. Cancer 95: $301-308$

Krammer PH (2000) CD95's deadly mission in the immune system. Nature 407: $789-795$

Lee Jr JT, McCubrey JA (2002) The Raf/MEK/ERK signal transduction cascade as a target for chemotherapeutic intervention in leukemia. Leukemia 16: $486-507$

Lee K, Esselman WJ (2002) Inhibition of PTPs by $\mathrm{H}(2) \mathrm{O}(2)$ regulates the activation of distinct MAPK pathways. Free Radic Biol Med 33: $1121-1132$

Lenormand P, Sardet C, Pages G, L'Allemain G, Brunet A, Pouyssegur, J (1993) Growth factors induce nuclear translocation of MAP kinases (p42mapk and p44mapk) but not of their activator MAP kinase kinase (p45mapkk) in fibroblasts. J Cell Biol 122: 1079-1088

Lutzker SG, Levine AJ (1996) A functionally inactive p53 protein in teratocarcinoma cells is activated by either DNA damage or cellular differentiation. Nat Med 2: 804-810

Nunez G, Benedict MA, Hu Y, Inohara N (1998) Caspases: the proteases of the apoptotic pathway. Oncogene 17: $3237-3245$

Palumbo C, van Roozendaal K, Gillis AJ, van Gurp RH, de Munnik H, Oosterhuis JW, van Zoelen EJ, Looijenga LH (2002) Expression of the PDGF alpha-receptor $1.5 \mathrm{~kb}$ transcript, OCT-4, and c-KIT in human normal and malignant tissues. Implications for the early diagnosis of testicular germ cell tumours and for our understanding of regulatory mechanisms. J Pathol 196: $467-477$

Persons DL, Yazlovitskaya EM, Cui W, Pelling JC (1999) Cisplatin-induced activation of mitogen-activated protein kinases in ovarian carcinoma cells: inhibition of extracellular signal-regulated kinase activity increases sensitivity to cisplatin. Clin Cancer Res 5: $1007-1014$ 
ERK I/2 mediates cisplatin-induced apoptosis in TGCT cell lines

S Schweyer et al

Peyssonnaux C, Eychene A (2001) The Raf/MEK/ERK pathway: new concepts of activation. Biol Cell 93: 53-62

Raabe T, Rapp UR (2002) KSR - a regulator and scaffold protein of the MAPK pathway. Sci STKE 136: PE28

Robbins DJ, Zhen E, Owaki H, Vanderbilt CA, Ebert D, Geppert TD, Cobb $\mathrm{MH}$ (1993) Regulation and properties of extracellular signal-regulated protein kinases 1 and 2 in vitro. J Biol Chem 268: 5097-5106

Salvesen GS, Dixit VM (1997) Caspases: intracellular signaling by proteolysis. Cell 91: $443-446$

Sorenson C, Eastman A (1988) Mechanism of cis-diamminedichloroplatinum(II)-induced cytotoxicity: role of G2 arrest and DNA double-strand breaks. Cancer Res 48: $6703-6707$

Thornberry NA, Lazebnik Y (1998) Caspases: enemies within. Science 281: $1312-1316$
Traverse S, Gomez N, Paterson H, Marshall C, Cohen P (1992) Sustained activation of the mitogen-activated protein (MAP) kinase cascade may be required for differentiation of PC12 cells. Comparison of the effects of nerve growth factor and epidermal growth factor. Biochem $J$ 288: $351-355$

Wang X, Martindale JL, Holbrook NJ (2000) Requirement for ERK activation in cisplatin-induced apoptosis. J Biol Chem 275: $39435-39443$

Wang X, Martindale JL, Liu Y, Holbrook NJ (1998) The cellular response to oxidative stress: influences of mitogen-activated protein kinase signalling pathways on cell survival. Biochem $J$ 333: $291-300$

Wyllie A, Kerr H, Currie AR (1980) Cell death: the significance of apoptosis. Int Rev Cytol 68: $251-306$ 